\title{
Big-Bang, Small-Bangs ou Fases: Estudo dos Aspectos Relacionados ao Modo de Início de Operação de Sistemas ERP
}

\author{
Cesar Alexandre de Souza \\ Ronaldo Zwicker
}

\begin{abstract}
Resumo
A escolha do modo de início de operação de um sistema integrado de gestão é importante decisão no seu projeto de implementação. Ela interfere de forma significativa na configuração do sistema, na alocação de recursos e no gerenciamento do projeto e de seus riscos. O modo de início de operação tem participação decisiva em todas as etapas do ciclo de vida do sistema. Este trabalho discute os diferentes modos de início de operação de sistemas ERP (Enterprise Resource Planning): big-bang, small-bangs e implementação em fases, incluindo suas vantagens e desvantagens. O trabalho também relata os resultados obtidos por meio de pesquisa exploratória realizada em 53 empresas brasileiras usuárias do sistema R/3 da SAP. A partir destes resultados, é sugerido como as características da empresa e do projeto influem na decisão pelo modo de início de operação e como elas se relacionam com o tempo necessário para a implementação do sistema.
\end{abstract}

Palavras-chaves: sistemas ERP; sistemas integrados de gestão; sistemas de informação.

\begin{abstract}
The choice of the implementation approach of an integrated management system is an important decision at the project implementation. It significantly influences the system configuration, allocation of resources, management of the project and its risks. The implementation approach mode plays a decisive role in all stages of the system's life cycle. Herein are addressed the different modes to start the operation of ERP (Enterprise Resource Planning) systems: big-bang, small-bangs and the phased implementation, as well as their advantages and disadvantages. The work also reports on the results achieved by an exploratory research carried out in 53 Brazilian companies, users of the R/3 system by SAP. These results infer how the company's and the project's characteristics affect the decision on the implementation approach and how they relate to the time required to implement the system.
\end{abstract}

Key words: ERP systems; integrated management systems; information systems. 


\section{INTRODUÇÃO}

A implementação de sistemas integrados de gestão (sistemas ERP) apresenta muitas dificuldades. Por exemplo, a necessidade de mudanças nos processos e na cultura da organização são questões bastante discutidas na bibliografia (Bancroft, Seip e Sprengel, 1998; Davenport, 1998; Bergamaschi e Reinhard, 2000; Souza e Zwicker, 2000). Outras questões igualmente importantes têm merecido menor atenção, como, por exemplo, a decisão crítica no processo de implementação que diz respeito ao modo de início de operação do sistema ERP (big-bang, small-bangs ou implementação em fases). Essa decisão depende de vários fatores tais como as limitações de recursos e de prazos, as características da equipe de projeto, o número de módulos que serão implementados, o número de localidades que serão atendidas e a natureza e a dimensão dos riscos que a empresa deseja assumir.

O texto que segue procura analisar os aspectos envolvidos com o modo de início de operação de sistemas ERP (Enterprise Resource Planning), mostrando sua relação com o ciclo de vida destes sistemas, identificando vantagens, desvantagens e fatores que devem ser considerados na escolha. São relatados os resultados de pesquisa exploratória realizada em 53 empresas que implementaram sistemas ERP (R/3 da SAP), procurando estabelecer relação entre fatores relevantes e a decisão tomada pela empresa para a implementação do seu sistema. Também são analisadas as conseqüências da escolha em relação ao tempo de implementação e de estabilização.

\section{Síntese Histórica dos Sistemas ERP}

Durante a segunda metade da década de 90, a implementação de sistemas ERP constituiu um dos principais focos de atenção relacionados com a utilização de Tecnologia da Informação (TI) nas empresas. Segundo Souza e Zwicker (2000), os sistemas ERP podem ser definidos como sistemas de informação integrados, adquiridos na forma de pacotes comerciais de software, com a finalidade de dar suporte à maioria das operações de uma empresa. Eles possuem características que, se consideradas em conjunto, os distinguem de outros sistemas de informação transacionais desenvolvidos internamente nas empresas e de outros tipos de sistemas comerciais. Em síntese os sistemas ERP: 
. são pacotes comerciais de software;

. incorporam modelos padrões de processos de negócios;

. constituem sistemas integrados de informações;

. utilizam um banco de dados corporativo;

· possuem grande abrangência funcional;

- requerem procedimentos de ajuste para que possam ser utilizados em determinada empresa (parametrizações e customizações).

Exemplos de sistemas ERP existentes no mercado são o R/3 da fornecedora alemã SAP, o Oracle Applications da norte-americana Oracle, o Magnus e o EMS da brasileira Datasul e o AP7 da brasileira Microsiga.

Em princípio, os sistemas ERP poderiam trazer inúmeros benefícios para as empresas. Entretanto rapidamente ficou evidente que a implementação de sistemas ERP era tarefa muito mais complexa do que a simples instalação de novo software na empresa. Tanto as empresas fornecedoras quanto as empresas de consultoria responsáveis pela implementação perceberam que a tarefa envolvia muitas dificuldades, em particular a mudança cultural de visão departamental da organização para a visão baseada em processos. Efetivamente muitos fatores interferem na implementação. Por exemplo, Bergamaschi e Reinhard (2000) apontam resultados de pesquisa que verificou quais fatores são essenciais para o sucesso de projetos de implementação de sistemas ERP. Os autores identificaram, entre outros fatores, a importância de se ter missões claras e definidas para o projeto de implementação, o apoio da alta administração, a existência de gerência de projetos competente e a realização de mudanças nos processos empresariais.

Também se passou a questionar se os propalados benefícios realmente se estariam concretizando após a entrada em operação dos sistemas. A questão do retorno do investimento foi considerada, mas sem que se pudesse em geral comprová-lo como imediatamente decorrente do uso destes sistemas. De qualquer forma, as empresas que os implementaram experimentaram inúmeras transformações e obtiveram muitos benefícios indiretos decorrentes da integração de seus processos. Por exemplo, Saccol et al. (2002) relatam os resultados de pesquisa realizada junto com amostra de 70 empresas brasileiras de grande porte e que avalia o impacto da utilização dos sistemas ERP sobre as variáveis estratégicas dessas organizações. Segundo os autores os resultados da pesquisa revelam poucas contribuições do sistema em relação a aspectos, estratégi- 
cos relacionados com clientes, competitividade e mercado. O ERP, entretanto, demonstrou agregar valor em relação a aspectos relacionados com fornecedores (por exemplo, relacionamento e monitoramento), produção (por exemplo, aumento de produtividade) e eficiência interorganizacional (por exemplo, facilitando a integração e comunicação entre diferentes unidades e com outras organizações).

Além do estudo da implementação e de seus benefícios também foi realizado no nosso ambiente um estudo crítico do fenômeno ERP, procurandose posicioná-lo em contexto mais amplo do que o restrito âmbito de aplicação operacional de sistemas de informação. Nesse sentido, Wood e Caldas (2000) abordam a questão do uso dos sistemas ERP como instrumentos de controle organizacional, baseando-se em modelo que analisa os fatores institucionais e políticos para explicar o fenômeno da utilização destes sistemas em empresas. Segundo os autores, esse modelo pode ser contraposto ao reducionismo tecnológico que caracteriza o discurso a respeito da implementação dos sistemas ERP. Mais do que uma tecnologia, um sistema ERP é artefato que causa impacto nas pessoas e sofre impacto por parte delas nas organizações.

Em que pesem todas as dificuldades enfrentadas, os sistemas ERP tornaram-se parte integrante e indissociável da operação de muitas empresas. É evidente, nos dias atuais, que o sucesso das empresas depende do bom funcionamento da tecnologia instalada. Em muitos casos é sobre esta base tecnológica que outras iniciativas foram ou estão sendo desenvolvidas, tais como o Customer Relationship Management (CRM), o Supply Chain Management (SCM) e os sistemas de inteligência empresarial (Business Intelligence).

\section{Ciclo de Vida de Sistemas ERP}

A implantação de um sistema ERP é realizada em etapas bem definidas. Souza e Zwicker (2001) apresentam um modelo para o ciclo de vida de sistemas ERP que inclui as etapas de decisão e seleção, implementação, estabilização e utilização. Estas grandes etapas e a sua relação temporal estão esquematizadas na Figura 1, que representa o ciclo de vida geral de sistemas ERP. 


\section{Figura 1: Ciclo de Vida de Sistemas ERP}

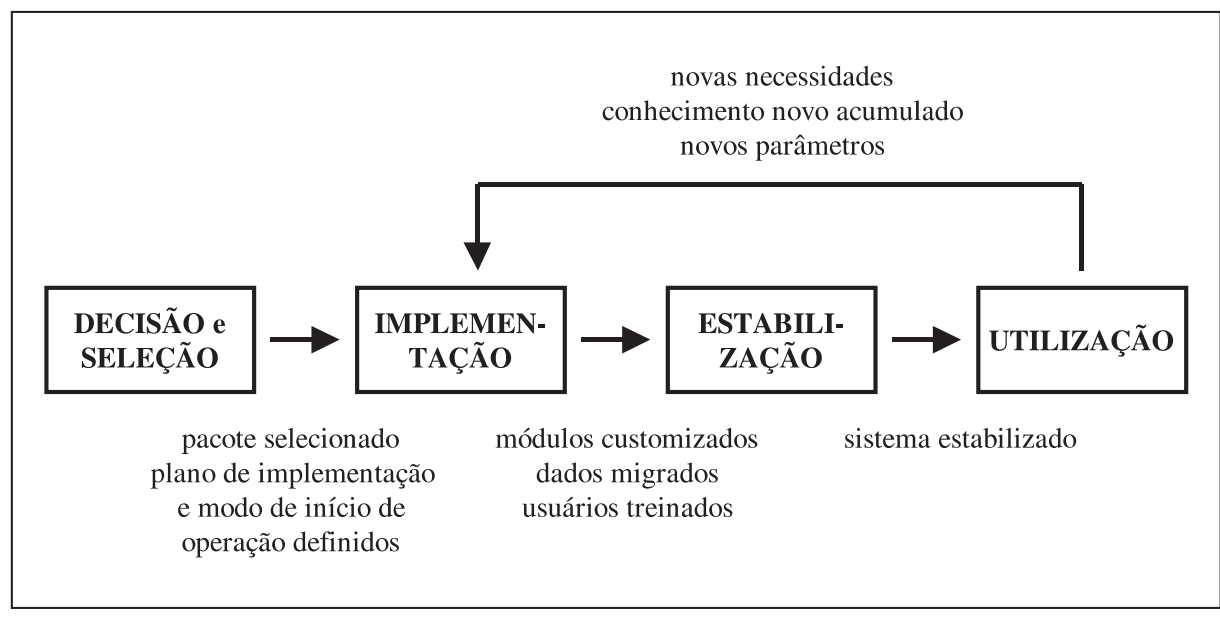

Na primeira etapa a empresa decide-se pela implementação de um sistema ERP como solução para as suas necessidades de informação e escolhe o fornecedor. Após a seleção do fornecedor, deve ser realizado o planejamento da implementação que engloba o estabelecimento dos objetivos e escopo do projeto, metas a serem cumpridas, métricas do projeto, definição de responsabilidades e a estratégia de implementação. A estratégia de implementação envolve a definição do modo de início de operação, das atividades que serão realizadas e do cronograma, o qual deve incluir considerações sobre prazos e recursos.

A implementação constitui a etapa seguinte do ciclo de vida e é definida como o processo pelo qual os módulos do sistema são colocados em funcionamento em uma empresa. Essa etapa envolve a adaptação dos processos de negócio ao sistema, a parametrização e eventual customização do sistema, a conversão e carga dos dados iniciais, a configuração do hardware e do software de suporte, o treinamento de usuários e gestores e a disponibilização de suporte e auxílio. Esta etapa contempla as tarefas que vão desde o término da elaboração do plano de implementação até o início da operação, quando o sistema ERP passa a ser o sistema de informação definitivo da empresa.

Após o início da operação começa uma etapa bastante crítica para o sucesso do projeto: a etapa de estabilização. Nessa etapa o sistema ERP torna-se um objeto concreto e passa a fazer parte do dia-a-dia da empresa e das pessoas. Esse é o momento em que a maior carga de energia, gerencial ou técnica, é necessária. Isto ocorre porque no início da operação do sistema (analogamente a qualquer sistema informatizado) evidenciam-se dificuldades de operação, falhas no treinamento, falhas de testes, erros em programas, novas customizações e 
problemas não previstos na etapa de implementação. Nesse momento a empresa já depende do sistema para as suas atividades, o que aumenta a pressão para que os problemas sejam rapidamente resolvidos. A duração desta etapa depende da empresa e gira em torno de oito semanas (Souza e Zwicker, 2001).

Finalmente, na etapa de utilização o sistema torna-se parte intrínseca das operações da empresa. Isto não significa que todas as suas possibilidades de uso tenham sido reconhecidas e estejam corretamente equacionadas. Este conhecimento só se estabelece após certo tempo de uso continuado da tecnologia e por meio de idéias que surgem durante o processo de utilização. Portanto a etapa de utilização realimenta a etapa de implementação com novas possibilidades e necessidades que podem ser resolvidas mediante implementação de novos módulos, novas parametrizações ou novas customizações.

\section{Modos de Início de Operação de Sistemas ERP}

Bancroft, Seip e Sprengel (1998), com base na análise de implementações de sistemas R/3 da SAP, afirmam que as primeiras decisões que a empresa deve tomar, após a escolha do pacote, devem dizer respeito a quais módulos serão implementados e em quais plantas ou localidades se dará esta implementação. Isso também se aplica a pacotes de outros fornecedores. Ao incluir a decisão sobre quando e em que ordem os módulos serão implementados, configura-se a determinação do modo de início de operação do sistema ERP na empresa. Essa escolha é decisiva para o projeto e influencia uma série de aspectos tais como prazos, recursos, requisitos de gerenciamento, entre outros.

Muitas empresas optam por implementar inicialmente um módulo ou grupos de módulos em uma ou mais localidades da empresa. Após esta implementação inicial o projeto prossegue com o próximo grupo de módulos e localidades. Essa é a alternativa em fases para o início de operação. Outra possibilidade é a implementação completa, em que todos os módulos contratados do pacote são implementados em todas as localidades simultaneamente, com a mesma data para início da operação. Essa alternativa é conhecida como big-bang. A alternativa em fases é mais segura, pois permite que a equipe de projeto aprenda com a experiência antes de colocar importantes processos da empresa no novo sistema. Entretanto, ela exige a construção de diversas interfaces do sistema antigo com novo, tarefa que consome recursos e cujos produtos são necessariamente descartados no final do projeto. 
Se a empresa possui mais de uma unidade de negócios ou localidade, há uma terceira possibilidade derivada da implementação em fases: o big-bang piloto ou small-bang. Nessa alternativa escolhe-se uma unidade de negócio ou localidade de menor porte ou importância para o início simultâneo (porém local) da operação. Dessa maneira é possível obter a experiência da implementação simultânea sem comprometer demais o negócio. O que segue ao small-bang tanto pode ser uma implementação simultânea tipo big-bang nas localidades restantes, como uma série de small-bangs ou implementações de módulos em fases.

A classificação das estratégias para início de operação de sistemas ERP pode ser feita segundo duas dimensões: a abrangência funcional e a abrangência geográfica. A abrangência funcional relaciona-se à quantidade de módulos que são implementados simultaneamente, enquanto a abrangência geográfica relacionase ao número de localidades ou unidades de negócio onde o sistema inicia a sua operação em um mesmo momento (vide Figura 2). Por exemplo, pode-se implementar o sistema ERP em algumas grandes etapas, cada uma envolvendo certo número de módulos simultâneos em determinado número de localidades simultâneas, o que caracteriza um modo de início de operação tipo small-bang (região A na Figura 2). Já a implementação de cada módulo simultaneamente em diversas localidades caracteriza uma implementação por fases em nível empresa (região B na Figura 2).

\section{Figura 2: Evolução do Risco de Interrupção das Operações}

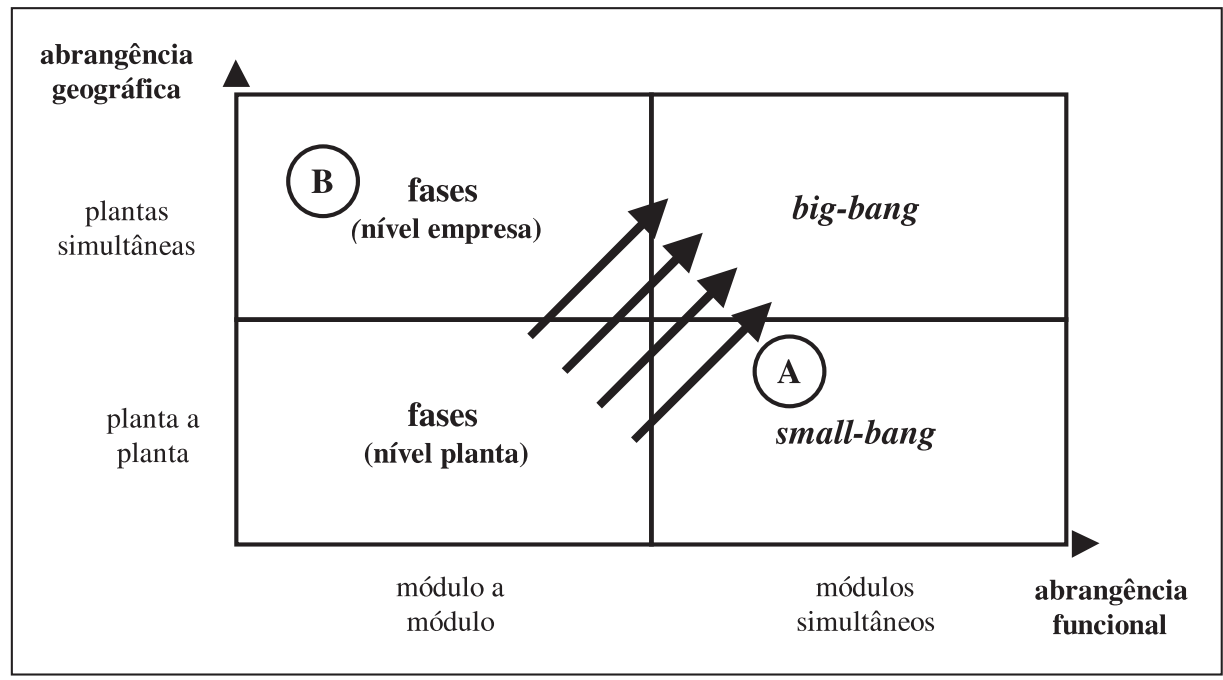

As setas no centro da Figura 2 sinalizam, para determinada empresa, o sentido geral de aumento do risco de interrupção das suas operações em função da adoção de diferentes estratégias de início de operação do seu sistema ERP. Esse 
risco, para determinada empresa, certamente está associado à quantidade de localidades que a empresa possui e à quantidade de módulos que serão implementados. A Figura 2 não compara o risco entre diferentes empresas; mas é justo supor que, em princípio, para determinada estratégia de início de operação, uma empresa que implementa maior número de módulos em maior número de localidades está sujeita a maior risco do que uma empresa implementando menor número de módulos em menos localidades.

\section{Conflitos do Modo de Início de Operação}

O modo de início de operação do sistema ERP tem grande influência nas etapas posteriores do ciclo de vida e principalmente na fase de estabilização. No caso big-bang, há clara distinção entre a etapa de implementação e a etapa de estabilização, o que não ocorre no caso da implementação em fases ou smallbangs. No caso big-bang, o início da operação do sistema corresponde à entrada em funcionamento de todos os módulos em todas as plantas; a atenção da equipe de projeto e da empresa volta-se para os esforços relacionados com a etapa de estabilização. Já na implementação em fases, coexistem módulos em etapas diferentes do ciclo de vida, o que traz problemas intrínsecos a este tipo de implementação.

Em princípio, o objetivo da etapa de implementação é o de ajustar o sistema e a empresa da melhor maneira possível, o que exige flexibilidade, testes constantes, mudanças em configurações, novas parametrizações e desenvolvimento de customizações. Por outro lado, o objetivo da etapa de estabilização é eliminar problemas no âmbito do ajuste predefinido na implementação. Isto gera conflitos, pois os sistemas ERP são sistemas integrados e a modificação em módulo em implementação (situação desejada na etapa de implementação) pode acarretar modificações em outros módulos já em utilização (situação não desejada nas etapas de estabilização e utilização). Esses conflitos ficam mais evidenciados no caso da implementação em fases e são agravados pelo fato de a etapa de estabilização iniciar-se com a entrada em operação do primeiro módulo e terminar apenas quando o último módulo implementado na última localidade da empresa se estabiliza.

\section{Vantagens e Desvantagens dos Modos de Início de Operação}

Embora considerada arriscada (Bancroft, Seip e Sprengel, 1998), a opção big- 
bang pode oferecer vantagens. Souza e Zwicker (2001) observaram, nos casos que analisaram, que o modo de início de operação em big-bang foi considerado importante motivador para o sucesso da implementação. Com esse modo de início estabelece-se na empresa o consenso de que não há possibilidade de retorno ao sistema anterior. A perspectiva de parada total das operações da empresa exerce ação favorável para a mudança e para a detecção de defeitos e conduz a um esforço conjunto para a resolução rápida dos problemas remanescentes na etapa de estabilização. O'Leary (2000) apresenta outras vantagens para a implementação em big-bang, tais como a eliminação da necessidade de construção de interfaces temporárias do sistema ERP com sistemas anteriores, uma melhor integração dos módulos implementados e menor prazo para implementação. O Quadro 1 resume estas observações.

\section{Quadro 1: Riscos e Vantagens do Modo de Início de Operação}

\begin{tabular}{|c|c|c|}
\hline & Riscos & Vantagens \\
\hline 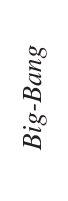 & $\begin{array}{l}\text { - aumenta risco de parada total da empresa } \\
\text { - difícil retorno ao sistema anterior } \\
\text { - exige grande esforço da equipe na etapa de } \\
\text { estabilização } \\
\text { - concentração de recursos durante o projeto }\end{array}$ & $\begin{array}{l}\text { - menor prazo de implementação } \\
\text { - maior motivação das pessoas } \\
\text { - elimina o desenvolvimento de interfaces } \\
\text { - gera senso de urgência que facilita o } \\
\text { estabelecimento de prioridades } \\
\text { - melhora a integração entre os vários módulos }\end{array}$ \\
\hline 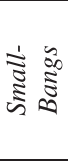 & $\begin{array}{l}\text { - aumenta risco de parada total da localidade } \\
\text { - difícil retorno ao sistema anterior } \\
\text { - é necessário o desenvolvimento de interfaces }\end{array}$ & $\begin{array}{l}\text { - maior motivação das pessoas } \\
\text { - gera senso de urgência que facilita o } \\
\text { estabelecimento de prioridades } \\
\text { - viabiliza o aprendizado a partir da } \\
\text { experiência }\end{array}$ \\
\hline 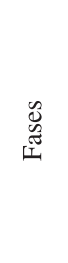 & $\begin{array}{l}\text { - é necessário o desenvolvimento de interfaces } \\
\text { - não há envolvimento de toda a empresa } \\
\text { - requisitos de módulos futuros são ignorados } \\
\text { - módulos em implementação acarretam } \\
\text { mudanças em módulos estabilizados } \\
\text { - implementação e estabilização simultâneas } \\
\text { - possível perda de foco do projeto } \\
\text { - maior movimentação de recursos humanos }\end{array}$ & $\begin{array}{l}\text { - menor risco de parada total da empresa } \\
\text { - pode-se voltar atrás em caso de problemas } \\
\text { - menor concentração de recursos ao longo do } \\
\text { projeto } \\
\text { - módulos em funcionamento geram confiança } \\
\text { no desenvolvimento dos subseqüentes } \\
\text { - menor intervalo de tempo entre a modelagem } \\
\text { e a utilização de cada módulo }\end{array}$ \\
\hline
\end{tabular}

Para O’Leary (2000) a escolha do modo de início de operação em princípio deveria ser feita com base na análise dos custos e benefícios de cada opção e considerando os riscos associados. No entanto, uma vez que custos e riscos são difíceis de mensurar, as empresas acabam tomando a decisão com base em outros aspectos. Assim, acabam prevalecendo na decisão aspectos relacionados com as características da organização e com a extensão da implementação.

As características da organização dizem respeito ao seu tamanho (faturamento, número de funcionários, volume de clientes) e complexidade (características do produto, do processo produtivo, do mercado atendido). Em princípio as organiza- 
ções menores e menos complexas correm menores riscos ao optar por uma implementação em big-bang e podem obter as vantagens associadas a essa estratégia. Quanto à extensão da implementação, é importante considerar a quantidade de módulos que serão implementados e o grau de customização que os módulos irão sofrer. O maior número de módulos e maior grau de customização aumenta a complexidade do projeto e, em conseqüência, a implementação em fases é preferível. Nos casos analisados por Souza e Zwicker (2001), embora não tenha sido possível estabelecer uma regra a respeito do modelo escolhido, pôde ser verificado que o big-bang foi utilizado nas empresas menores ou naquelas em que havia restrições de prazo muito claras. Nas empresas maiores a implementação em fases teve preferência e o big-bang foi eventualmente considerado totalmente inviável. O Quadro 2 sintetiza esta breve discussão.

\section{Quadro 2: Aspectos da Escolha do Modo de Início de Operação}

- Objetivos do projeto

Vantagens e desvantagens de cada alternativa

Riscos associados com cada alternativa

Complexidade da empresa e de seus processos

Tamanho da empresa

Características dos produtos e dos processos

Características do mercado e dos negócios

Complexidade do projeto

Número de módulos a serem implementados

Grau de customização

Abrangência geográfica e número de plantas

Configuração empresarial das localidades (filiais e grupo de empresas)

Restrições do projeto

Prazos

Recursos financeiros

Limitações técnicas e capacitação dos recursos humanos

\section{Metodologia da Pesquisa}

O trabalho relata os resultados de pesquisa realizada em 2001 pelos autores em parceria com a ASUG-Brasil (grupo de usuários da SAP no Brasil). A pesquisa envolveu a coleta de dados mediante questionário enviado aos 254 membros da ASUG, com retorno de 55 questionários (cerca de 21\% de respostas). Os questionários foram respondidos entre janeiro e fevereiro de 2001. Dos 55 questionários respondidos, 53 foram considerados adequados para este estudo. Desse total, 6 foram respondidos por empresas que ainda não haviam iniciado a operação do 
sistema, sendo nestes casos utilizada a previsão fornecida para o término do projeto como informação da sua duração efetiva.

O questionário enviado caracterizava a empresa e o sistema implementado e envolvia questões específicas sobre a estratégia de implementação adotada e sobre o tempo de duração do projeto. A partir das respostas, foi realizada uma análise exploratória, focalizando a questão da estratégia de implementação (bigbang, small-bangs ou fases) e buscando verificar se e como esta estratégia mantém relação com as características das empresas e dos projetos de implementação. Após essa análise, a pesquisa procurou verificar se o modo de implementação escolhido e as características influenciaram o tempo de duração do projeto. As análises estatísticas foram realizadas com o auxílio do software SPSS for Windows 10.0.

\section{Análise Descritiva e Exploratória dos Dados}

\section{Caracterização da Amostra}

A distribuição das empresas na amostra indica que 71\% delas adotaram o bigbang, $15 \%$ o small-bang, $8 \%$ a implementação em fases e as restantes adotaram outros procedimentos. A maioria das empresas eram industriais (78\%) e as restantes estavam repartidas uniformemente entre os setores de serviços, utilidades e comércio. O número de empresas que utilizaram a opção big-bang é bastante representativo, superando a expectativa inicial de que esta seria a escolha menos utilizada pelas empresas. A predominância de empresas industriais ainda é característica marcante do mercado de sistemas ERP. Com relação à nacionalidade, as empresas estão igualmente divididas entre a origem nacional e estrangeira.

Na Tabela 1 estão apresentadas estatísticas das variáveis métricas de interesse observadas e o número de casos que continham informação sobre cada variável. O coeficiente de assimetria foi calculado utilizando-se a medida de skewness fornecida pelo SPSS dividida por seu desvio padrão. Consideram-se simétricas as distribuições em que este valor se situa na faixa entre -1,96 e 1,96 (Hair et al., 1998). Apenas a idade do projeto (IDADEPRJ) pôde ser considerada simétrica, sendo as demais positivamente assimétricas, o que indica concentração dos valores no início das distribuições. Apesar disso, a mediana permite boa análise das características das empresas pesquisadas e de seus projetos. São em geral empresas de porte com faturamento ao redor de US\$300 milhões e com mais de 1.500 funcionários. Os projetos implementados referem-se em geral a 6 módulos 
(o coração do R/3 é composto de 5 módulos) em 4 plantas e com um total de 300 usuários. A mediana do porte do projeto (MODPLAN) é 18, menor do que a simples multiplicação de módulos por plantas $(6 \times 4=24)$. Isso indica alguns módulos são implementados de maneira centralizada (em algumas plantas), enquanto que outros de maneira descentralizada (em todas as plantas).

Tabela 1: Características das Variáveis de Interesse

\begin{tabular}{|l|l|r|r|r|r|r|r|r|}
\hline $\begin{array}{c}\text { nome da } \\
\text { variável }\end{array}$ & \multicolumn{1}{|c|}{ descrição (veja Quadro 3) } & média & $\begin{array}{c}\text { desvio } \\
\text { padrão }\end{array}$ & $\begin{array}{c}\text { media- } \\
\text { na }\end{array}$ & $\begin{array}{c}\text { menor } \\
\text { valor }\end{array}$ & $\begin{array}{c}\text { maior } \\
\text { valor }\end{array}$ & $\begin{array}{c}\text { coef. } \\
\text { assim. }\end{array}$ & $\begin{array}{c}\text { no. de } \\
\text { casos }\end{array}$ \\
\hline TIMPLANT & tempo de duração do projeto & 16,3 & 11,2 & 12 & 3 & 48 & 4,4 & 53 \\
\hline TESTABLZ & tempo de estabilização & 10,5 & 9,0 & 8 & 1 & 48 & 3,9 & 53 \\
\hline IDADEPRJ & idade do projeto & 33,0 & 14,3 & 33 & 4 & 71 & 1,3 & 53 \\
\hline FATBR & faturamento em US\$ milhões & 530,0 & 858,0 & 300 & 20 & 5000 & 10,1 & 47 \\
\hline FUNCBR & no. de funcionários & 4817,0 & 8082,0 & 1725 & 60 & 40000 & 8,6 & 50 \\
\hline USUBRA & no. de usuários do R/3 & 487,0 & 610,0 & 300 & 15 & 3000 & 7,8 & 53 \\
\hline PLANTAS & no. de plantas em implantação & 6,1 & 7,3 & 4 & 1 & 32 & 7,5 & 50 \\
\hline MÓDULOS & no. de módulos implementados & 6,8 & 2,2 & 6 & 3 & 13 & 2,3 & 53 \\
\hline MODPLAN & porte do projeto & 32,0 & 41,4 & 18 & 4 & 256 & 11,3 & 50 \\
\hline QTSERVDR & no. de servidores & 4,3 & 4,5 & 3 & 1 & 27 & 9,9 & 53 \\
\hline TOTPROGS & programas desenvolvidos & 364,0 & 428,0 & 253 & 31 & 2131 & 7,9 & 45 \\
\hline FUNCTI & funcionários na equipe de TI & 33,4 & 49,2 & 17 & 2 & 283 & 9,9 & 42 \\
\hline
\end{tabular}

\section{Quadro 3: Descrição Adicional das Variáveis}

\begin{tabular}{|l|l|}
\hline \multicolumn{1}{|c|}{ variável } & \multicolumn{1}{c|}{ descrição } \\
\hline TIMPLANT & $\begin{array}{l}\text { Tempo de duração do projeto em meses desde seu início até o início da operação do } \\
\text { último módulo. Nos projetos em andamento foi usada a previsão de término do projeto. }\end{array}$ \\
\hline TESTABLZ & $\begin{array}{l}\text { Tempo de estabilização do sistema em semanas após o início da sua operação. Apenas } \\
\text { se aplica às situações de big-bang. }\end{array}$ \\
\hline IDADEPRJ & $\begin{array}{l}\text { Medida da idade do projeto. É o número de meses transcorridos desde o início do } \\
\text { projeto até a data da realização da pesquisa (janeiro de 2001). }\end{array}$ \\
\hline TOTPROGS & $\begin{array}{l}\text { Total de programas externos (inclusive em linguagem ABAP) e relatórios (inclusive } \\
\text { gerados por gerador) que a empresa desenvolveu até a data de realização da pesquisa. }\end{array}$ \\
\hline MODPLAN & $\begin{array}{l}\text { Medida do porte do projeto. As empresas informaram para cada módulo o número de } \\
\text { plantas onde este módulo era utilizado. A variável é a somatória destes valores. }\end{array}$ \\
\hline
\end{tabular}

O Gráfico 1 dá uma idéia da dispersão dos projetos, divididos em tipo big-bang e outros, de acordo com o número de usuários e porte do projeto (os eixos estão em escala logarítmica). Os quatro maiores projetos indicados no gráfico são referentes a este elenco: uma indústria de autopeças (caso 19, 3.000 usuários, 12 módulos, 7 plantas); uma indústria química (caso 50, 2.600 usuários, 8 módulos, 32 plantas); 
uma empresa alimentícia (caso 51, 1.450 usuários, 5 módulos, 29 plantas); e uma indústria de fumo (caso 25, 1.200 usuários, 8 módulos, 27 plantas). Desses casos apenas a indústria química usou a estratégia big-bang e teve o maior tempo de duração de projeto da amostra para esse tipo de modo de início (21 meses). O caso 24 é uma indústria química nacional de médio porte que implementou o R/3 em small-bangs para um total de 17 usuários em 5 plantas. O Gráfico 2 mostra a distribuição dos tempos de implementação dos projetos estudados.

\section{Gráfico 1: Dispersão dos Projetos por Número de Usuários e Porte}

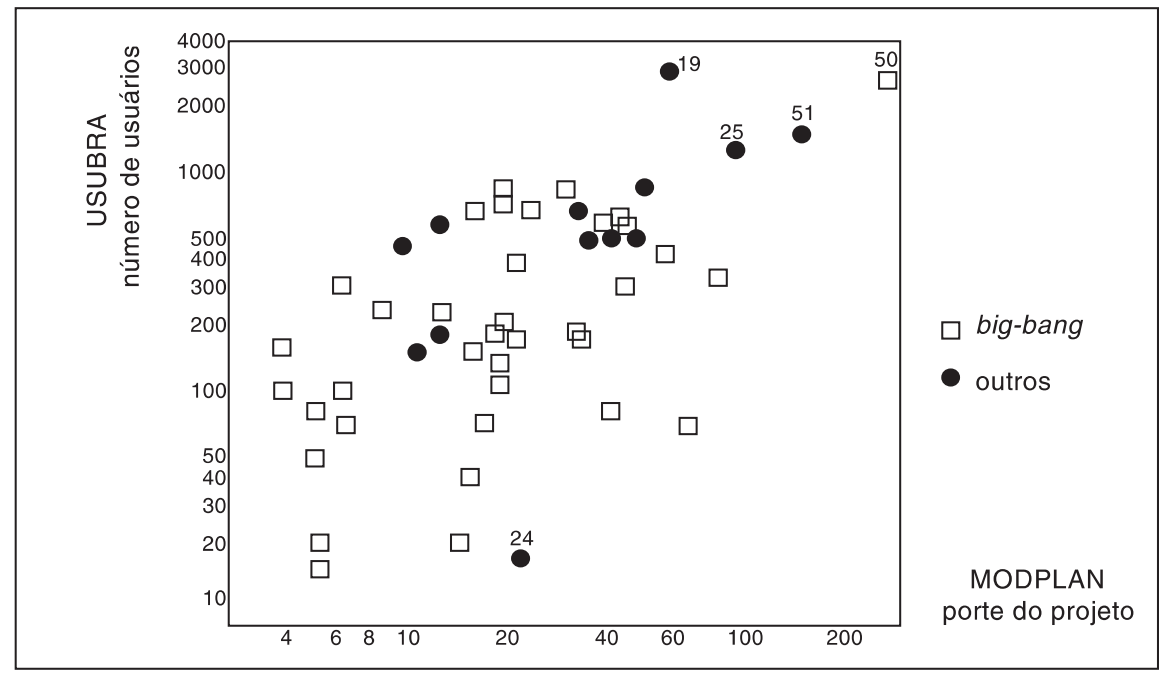

Gráfico 2: Distribuição dos Tempos de Implantação dos Projetos

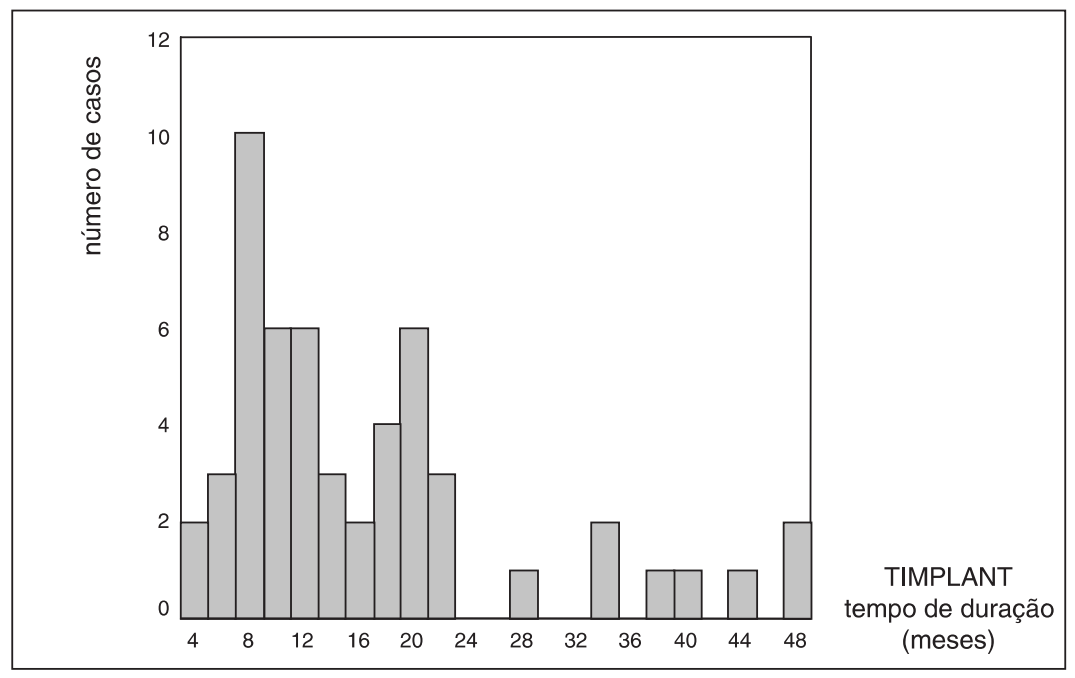


Quanto ao tempo de estabilização (TESTABLZ) verificou-se que 4 empresas indicaram valores muito acima das demais (40, 30, 28 e 26 semanas). Dois fatores parecem ter contribuído para essa discrepância: (1) o conceito de estabilização depende do nível de problemas que a empresa considera como normal; (2) alguns módulos estabilizam mais rapidamente do que outros. Uma das empresas informou que o tempo necessário para recuperar a confiabilidade no controle de estoques e custos de produção foi de 48 semanas, enquanto outros módulos tiveram estabilização muito mais rápida (4 semanas). Neste caso foi considerado o tempo médio de 26 semanas. Eliminando essas observações, obtém-se o valor médio de 8 semanas semelhante ao relatado por Souza e Zwicker (2001). Notese que esta questão foi respondida apenas por empresas com implementação em big-bang.

\section{Relações Envolvendo o Modo de Início de Operação}

Para a verificação da relação entre o modo de início de operação e as demais variáveis, dividiu-se a amostra em dois grupos: big-bang e outros. Essa divisão foi feita porque o número de casos pertencentes a cada uma das outras categorias (small-bangs e fases) ficou reduzido em comparação ao número de casos que utilizaram big-bang. Como se verá adiante, essa divisão mostrou consistência nas análises realizadas.

Para as variáveis nominais (nacionalidade e setor de atividade da empresa), verificou-se por meio do teste do qui-quadrado se havia diferença quanto à constituição dos grupos big-bang e outros. Não foram detectadas diferenças significativas de proporção entre empresas que utilizaram o modo big-bang ou outros, com base em nacionalidade ou setor de atividade. Para a verificação de diferenças nas variáveis métricas entre os dois grupos, foi utilizado o teste t, cujos resultados são apresentados na Tabela 2.

O teste $t$ é bastante robusto, mas depende da simetria da distribuição das variáveis e da igualdade de variâncias entre os grupos. Nos casos em que a variância dos grupos se mostrou diferente (com base no teste de Levene, realizado pelo SPSS), são também apresentados os resultados do teste $t$, com base nas variáveis transformadas, utilizando-se o logaritmo na base $e(I n)$ para compensar a assimetria. Seu uso não alterou significativamente as conclusões, havendo até mesmo reforço das diferenças encontradas.

A diferença no tempo de implementação entre os dois grupos é estatisticamente significativa com média de 11,9 meses para as empresas que implementaram em big-bang e média de 27,3 meses para as que implementaram em outros modos. As diferenças observadas nas variáveis FATBR e FUNCBR, que representam o 
tamanho da empresa, ficaram acima da significância de 0,05, embora dentro da faixa de significância 0,10. No caso destas variáveis a transformação permitiu a validação estatística das diferenças. Já a variável USUBRA mostrou diferença significativa, enquanto as variáveis MODPLAN e MODULOS não. As demais variáveis (não incluídas na tabela) não apresentaram diferenças significativas.

\section{Tabela 2: Resultados do Teste $t$ para Modos Big-Bang e Outros}

\begin{tabular}{|l|r|r|r|r|r|r|r|r|}
\hline & \multicolumn{5}{|c|}{ variável original } & \multicolumn{3}{c|}{ variável transformada (ln) } \\
\hline variável & $\begin{array}{c}\text { significân- } \\
\text { cia }\left(^{*}\right)\end{array}$ & valor de $t$ & $\begin{array}{c}\text { signifi- } \\
\text { cância de } \\
t\end{array}$ & $\begin{array}{c}\text { valor } \\
\text { médio } \\
\text { big-bang }\end{array}$ & $\begin{array}{c}\text { valor } \\
\text { médio } \\
\text { outros }\end{array}$ & $\begin{array}{c}\text { significân- } \\
\text { cia }\left(^{*}\right)\end{array}$ & valor de $t$ & $\begin{array}{c}\text { signifi- } \\
\text { cância de } \\
t\end{array}$ \\
\hline TIMPLANT & 0,000 & $-4,070$ & $\mathbf{0 , 0 0 1}$ & 11,9 & 27,3 & 0,199 & $-4,789$ & $<\mathbf{0 , 0 0 1}$ \\
\hline FATBR & 0,001 & $-1,809$ & 0,094 & 330,6 & 1053,0 & 0,750 & $-2,785$ & $\mathbf{0 , 0 1 7}$ \\
\hline FUNCBR & 0,000 & $-2,097$ & 0,054 & 2500,0 & 10225,0 & 0,228 & $-2,811$ & $\mathbf{0 , 0 0 7}$ \\
\hline USUBRA & 0,028 & $-2,149$ & $\mathbf{0 , 0 4 6}$ & 353,3 & 824,5 & 0,549 & $-2,671$ & $\mathbf{0 , 0 1 0}$ \\
\hline MODPLAN & 0,689 & $-1,031$ & 0,308 & 28,4 & 42,1 & -- & -- & -- \\
\hline MODULOS & 0,544 & $-1,079$ & 0,286 & 6,5 & 7,3 & -- & -- & -- \\
\hline IDADEPRJ & 0,366 & $-2,335$ & $\mathbf{0 , 0 2 4}$ & 30,2 & 40,0 & -- & -- \\
\hline
\end{tabular}

Nota: (*) O teste de Levene verifica a igualdade das variâncias da variável nos dois grupos. A hipótese nula é a igualdade entre as variâncias. Se as variâncias não são iguais, então é apresentado o valor do t corrigido.

De acordo com estes resultados, pode-se concluir que o big-bang foi utilizado em projetos da amostra com menor número de usuários e menor tempo de implementação. Aparentemente o big-bang está relacionado a empresas menores, de acordo com as variáveis quantidade de funcionários e faturamento. Também é sugestiva a diferença apresentada em relação à idade do projeto, que teve média de 30,2 meses para o grupo big-bang e de 40,0 meses para o grupo outros, com significância de 0,024. Isso indica que os projetos big-bang da amostra são mais recentes do que os demais.

\section{Relações que Envolvem a Natureza das Empresas}

A Tabela 3 apresenta os resultados do teste $t$ para a verificação da relação entre TIMPLANT e IDADEPRJ com a nacionalidade e o setor das empresas. Como se pode observar, TIMPLANT não apresentou diferença significativa entre as empresas brasileiras e as estrangeiras, nem entre as empresas do setor industrial e as demais. Entretanto IDADEPRJ foi significativamente maior nas empresas estrangeiras da amostra, o que indica que elas foram as primeiras a implementar o R/3. O número de módulos implementados mostrou diferença significativa em relação ao setor de atividade, com maior quantidade de módulos para as empresas industriais. 
As demais variáveis não apresentaram diferenças significativas entre as empresas brasileiras e estrangeiras e as do setor industrial e de outros setores.

\section{Tabela 3: Resultados do Teste $t$ para Nacionalidade e Setor de Atividade}

\begin{tabular}{|c|c|r|r|r|r|r|r|r|}
\hline & \multicolumn{4}{|c|}{ nacionalidade da empresa } & \multicolumn{4}{c|}{ setor de atividade } \\
\hline variável & $\begin{array}{c}\text { valor } \\
\text { de } t\end{array}$ & $\begin{array}{c}\text { significân- } \\
\text { cia de } t\end{array}$ & $\begin{array}{c}\text { média } \\
\text { nacionais }\end{array}$ & $\begin{array}{c}\text { média } \text { es- } \\
\text { trangeiras }\end{array}$ & $\begin{array}{c}\text { valor } \\
\text { de } t\end{array}$ & $\begin{array}{c}\text { significân- } \\
\text { cia de } t\end{array}$ & $\begin{array}{c}\text { média } \text { in- } \\
\text { dustriais }\end{array}$ & $\begin{array}{c}\text { média } \\
\text { outras }\end{array}$ \\
\hline TIMPLANT & $-1,585$ & 0,119 & 13,8 & 18,7 & 1,085 & 0,283 & 17,1 & 13,0 \\
\hline IDADEPRJ & $-2,011$ & $\mathbf{0 , 0 5 0}$ & 29,1 & 36,7 & 1,662 & 0,108 & 34,2 & 28,2 \\
\hline MÓDULOS & $-0,078$ & 0,938 & 6,7 & 6,7 & 2,878 & $\mathbf{0 , 0 0 6}$ & 7,2 & 5,2 \\
\hline
\end{tabular}

\section{Relações entre Variáveis}

Com a finalidade de analisar as relações entre as variáveis métricas estudadas foram calculados os coeficientes de correlação linear de Pearson. Os valores com significância menor ou igual a 0,5 estão apresentados na Tabela 4. A transformação logarítmica ( $l n$ ) das variáveis para compensar a assimetria não alterou significativamente a interpretação dos coeficientes de correlação, sendo mantidas as variáveis originais. A exceção foi a variável USUBRA que, transformada, recebeu a denominação LNUSUBRA e está incluída na tabela.

\section{Tabela 4: Coeficientes de Correlação de Pearson entre as Variáveis Métricas}

\begin{tabular}{|c|c|c|c|c|c|c|c|c|c|}
\hline & 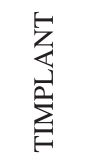 & 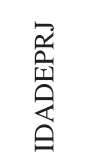 & 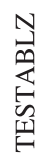 & 㟧 & $\begin{array}{l}\text { 品 } \\
\text { 苔 } \\
\text { 足 }\end{array}$ & 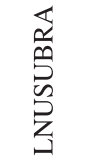 & 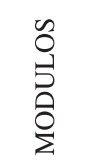 & 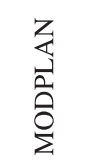 & 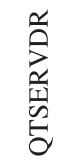 \\
\hline \multicolumn{10}{|l|}{ TIMPLAN } \\
\hline IDADEPR &, $618 * *$ & & & & & & & & \\
\hline \multicolumn{10}{|l|}{ TESTABL } \\
\hline FATBR &, $525 * *$ & & & & & & & & \\
\hline FUNCBR & $328^{*}$ & & & ,782** & & & & & \\
\hline LNUSUB &, $558 * *$ &, $437 * *$ & & ,421* &, $369 * *$ & & & & \\
\hline MODULO &, $477 * *$ &, $410 * *$ & & & &, $484 * *$ & & & \\
\hline MODPLA & & & &, $383 * *$ & &, $519 * *$ & & & \\
\hline QTDSVD &, $421^{* *}$ & & &, $483 * *$ & & ,458** &, $401 * *$ &, $649 * *$ & \\
\hline TOTPRO &, $449 * *$ & ,344* & & & ,388* &, $469 * *$ & &, $568 * *$ & \\
\hline FUNCTI &, $488 * *$ & & &, $902 * *$ &, $742 * *$ &, $400 * *$ & & & $474 * *$ \\
\hline
\end{tabular}

Nota: $(*)$ correlação significativa em nível 0,05; (**) correlação significativa em nível 0,01. 
A duração do projeto TIMPLANT mostrou-se fortemente correlacionada à idade do projeto IDADEPRJ. Essa é uma constatação interessante que mostra uma redução no tempo dos projetos de implementação mais recentes. TIMPLANT também apresentou correlação com FATBR e em menor grau com FUNCBR, o que aponta aumento no tempo de implementação em projetos de empresas maiores. TIMPLANT mostrou correlação com o tamanho do projeto, indicado por LNUSUBRA, e correlação moderada com MODULOS.

IDADEPRJ está correlacionada ainda com MODULOS e a quantidade total de programas desenvolvidos TOTPROGS. Isso sugere que as empresas continuam implementando módulos menores ou menos abrangentes, complementando o sistema com o passar do tempo. Como era esperado, o número de usuários mostrou forte correlação com o porte do projeto. Analogamente o número de servidores está bastante correlacionado com MODPLAN e LNUSUBRA, em vista da carga de processamento envolvida em sistemas ERP. TOTPROGS está correlacionado à MODPLAN e LNUSUBRA e não diretamente ao número de módulos, como seria de se esperar.

Outra constatação é o fato de o tempo de estabilização TESTABLZ não estar correlacionado com nenhum outro fator (para os casos big-bang). Provavelmente isto decorre do fato de que após o início da operação em bigbang não há alternativa para a empresa senão estabilizar o sistema em curto prazo, porquanto, caso contrário, suas operações serão prejudicadas. Isto está de acordo com o senso de urgência e motivação atribuídos à implementação em big-bang.

\section{Relações que Envolvem o Número de Usuários e o Tempo de Implementação}

Conforme observado, o número de usuários mostrou-se significativamente diferente entre os grupos big-bang e outros. O Gráfico 3 mostra a relação entre USUBRA e TIMPLANT e a opção de modo de início. Nele é possível verificar que todas as empresas com menos de 150 usuários optaram pela utilização do big-bang (exceto o caso 24). Acima desse número existem projetos big-bang e outros, sendo maiores os tempos de implementação dos projetos outros.

O Gráfico 3 esclarece a distribuição de TIMPLANT apresentada no Gráfico 2 e a composição dos projetos estudados, pois é possível observar a existência de três grupos de empresas: as empresas big-bang com até 200 usuários, as empresas 
big-bang com mais de 200 usuários e as empresas que implementaram em fases ou small-bangs. Projetando os pontos sobre o eixo de TIMPLANT, podemos ver que há concentração do primeiro grupo em torno do valor de 8 meses; do segundo grupo em torno do valor de 20 meses; e do terceiro grupo (mais disperso) entre 12 e 53 meses. Isso reflete exatamente a distribuição apresentada no Gráfico 2.

\section{Gráfico 3: Dispersão dos Projetos por Duração e Número de Usuários}

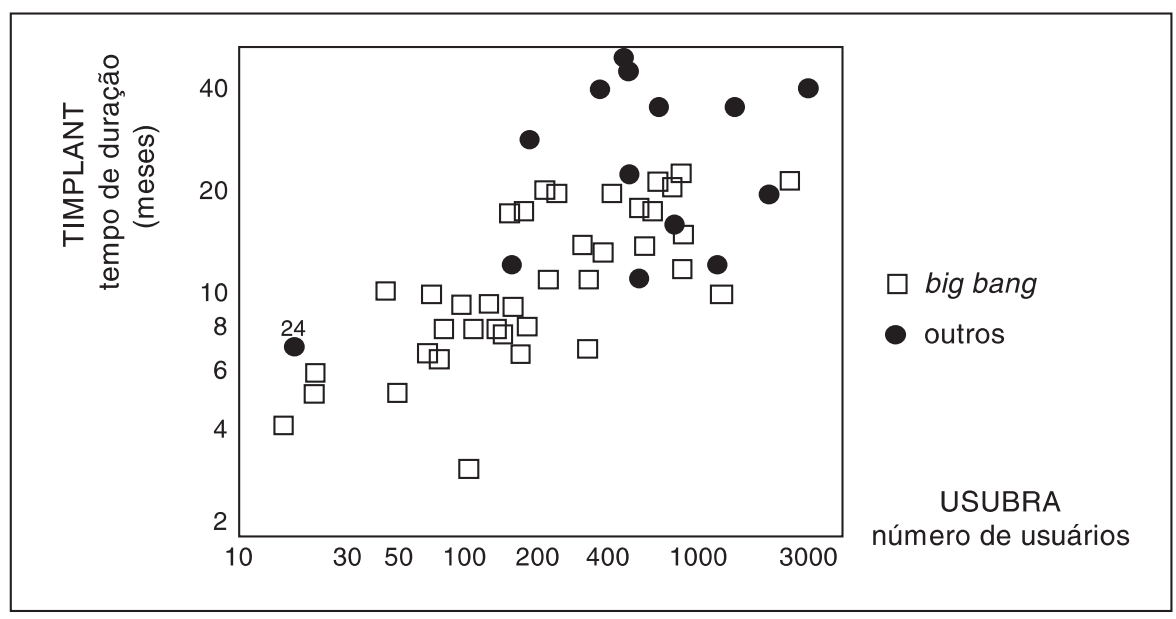

Também é possível observar que, no caso das empresas big-bang, há correlação mais evidente entre USUBRA e TIMPLANT do que no caso das outras empresas (coeficiente de correlação 0,700 com significância de 0,001). Dividindo-se os projetos big-bang em dois grupos (até 200 usuários e acima de 200 usuários) obtiveram-se os resultados para testes t das variáveis em estudo, apresentados na Tabela 5 (com diferenças significativas). Pelos resultados, essa divisão dos projetos big-bang mostrou-se bastante consistente, apresentando grupos diferenciados por FATBR e IDADEPRJ. O grupo até 200 usuários é composto por empresas menores e com implementações mais recentes.

\section{Tabela 5: Comparação dos Dois Grupos Big-Bang}

\begin{tabular}{|l|c|r|r|r|}
\hline \multicolumn{1}{|c|}{ Variáveis } & valor de t & $\begin{array}{c}\text { significância } \\
\text { de } \mathrm{t}\end{array}$ & $\begin{array}{c}\text { média: } \\
\text { até 200 usuários }\end{array}$ & $\begin{array}{c}\text { média: mais de } \\
\text { 200 usuários }\end{array}$ \\
\hline TIMPLANT & 4,568 & $<0,001$ & 8,9 & 15,6 \\
\hline IDADEPRJ & 2,997 & 0,005 & 25,3 & 36,3 \\
\hline FATBR & 3,751 & 0,002 & 139,4 & 603,6 \\
\hline TOTPROGS & 3,111 & 0,003 & 135,1 & 490,0 \\
\hline MODULOS & 3,585 & 0,001 & 5,8 & 7,7 \\
\hline
\end{tabular}


A diferença na quantidade de programas desenvolvidos e de módulos implementados pode estar relacionada ao fato de serem os projetos mais simples no primeiro grupo ou, ainda, à adoção pelas empresas menores de soluções mais padronizadas.

\section{A 'Aprendizagem do Mercado'}

Dos resultados apresentados ressalta-se a relação entre a idade do projeto e o tempo de implementação. O Gráfico 4 mostra a relação entre as duas variáveis para projetos big-bang e outros. A análise do gráfico indica uma evolução no tempo da direita para a esquerda e um efeito de redução de tempo de cima para baixo. Observa-se que mais recentemente os projetos tendem a adotar a estratégia big-bang e, simultaneamente, há tendência de redução do tempo de implementação TIMPLANT.

\section{Gráfico 4: Dispersão dos Projetos por Duração e Idade}

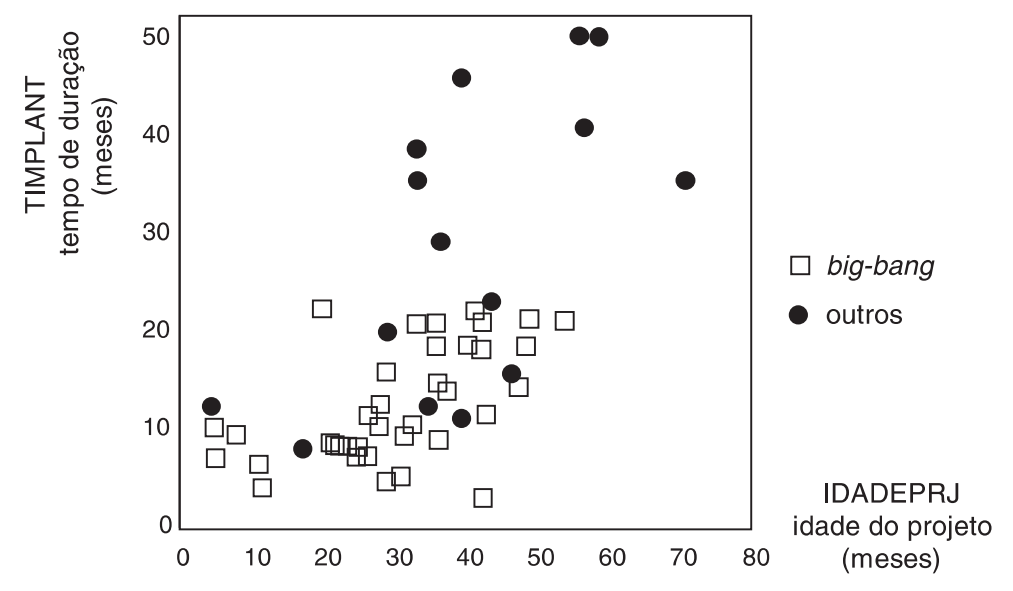

Isto pode ocorrer porque os projetos estão efetivamente ficando mais rápidos, ou ainda porque as empresas maiores, com projetos maiores, implementaram o R/3 antes das demais. Para melhor analisar a tendência observada, são apresentadas na Tabela 6 as correlações totais e parciais entre TIMPLANT, IDADEPRJ e LNUSUBRA, essa última escolhida como indicadora do porte do projeto. A correlação parcial é a correlação entre duas variáveis, quando é excluído o efeito das demais (Hair et al., 1998). 


\section{Tabela 6: Correlações entre Variáveis}

\begin{tabular}{|l|r|r|r|r|}
\hline \multirow{2}{*}{} & \multicolumn{2}{|c|}{ todos os projetos } & \multicolumn{2}{c|}{ somente big-bang } \\
\cline { 2 - 5 } & LNUSUBRA & IDADEPRJ & LNUSUBRA & IDADEPRJ \\
\hline $\begin{array}{l}\text { IDADEPRJ } \\
\text { Correlação total }\end{array}$ &, $4373^{* *}$ & &, $4300^{* * *}$ & \\
\hline TIMPLANT &, $5583^{* *}$ &, $6180^{* *}$ &, $7000^{* *}$ &, $5740^{* *}$ \\
Correlação total &, $4075^{* *}$ &, $5010^{* *}$ &, $6126^{* *}$ &, $4233^{* *}$ \\
Correlação parcial &, & & & \\
\hline
\end{tabular}

Como já comentado anteriormente, a correlação entre IDADEPRJ e LNUSUBRA indica que os projetos mais recentes são menores, o que também foi mostrado no item anterior em relação aos projetos big-bang. A correlação parcial entre TIMPLANT e IDADEPRJ permaneceu significativa, o que indica que projetos mais recentes efetivamente são mais rápidos, mesmo retirado o efeito da redução de usuários nos projetos mais novos. Esse efeito é maior, se considerados todos os projetos, não somente os projetos big-bang. A correlação parcial entre TIMPLANT e LNUSUBRA também indica que, descontada a idade do projeto, projetos com menos usuários têm tempos menores para implementação. No caso dessa correlação, o efeito é maior nos projetos bigbang. Assim, a redução do tempo dos projetos mais recentes está associada, em parte, à redução no tamanho e, em parte, à redução do tempo necessário à implementação.

Uma possível explicação para a parcela de redução no tempo de implementação não explicada pela redução no tamanho dos projetos seria a aprendizagem das empresas de consultoria que implementam o $\mathrm{R} / 3$, dos profissionais do mercado e do próprio fornecedor em relação aos projetos de implementação e ao funcionamento do pacote. A redução do tempo também pode ser atribuída a melhorias introduzidas no próprio pacote ao longo do tempo, principalmente no que se refere a aspectos de localização (adaptação do R/3 à legislação brasileira) e que diminui a necessidade de customizações. A existência de mais informações sobre empresas que implementaram, bem como a existência de mecanismos de troca de experiências, tais como grupos de usuários, também podem explicar essa redução no tempo. Todo esse conjunto de fatores poderia ser interpretado como aprendizagem do mercado em relação ao R/3. Entretanto não é possível detectar a influência ou mesmo a existência desse aprendizado apenas com base nos dados da pesquisa realizada. 


\section{Conclusões}

Inicialmente deve ser observado que, ao generalizar os resultados apresentados, é necessário levar em consideração que a amostra não é aleatória e consiste apenas em parte dos membros da ASUG-Brasil (cerca de 21\%). Entretanto a amostra provavelmente representa bem esta população, bem como a totalidade das empresas usuárias brasileiras do sistema R/3. Conforme dados de abril de 2002 do site da SAP, existiam cerca de 380 usuários do R/3 no Brasil. A amostra representa cerca $14 \%$ desse total.

A pesquisa realizada permitiu observar alguns aspectos dos projetos de implementação do sistema R/3 no Brasil. Inicialmente constatou-se que há número expressivo de projetos que utilizam o modo de início de operação em bigbang e que, em geral, estes projetos estão ligados a empresas com menor número de usuários. Também se verificou que o tempo de implementação destes projetos é menor. Observou-se ainda o incremento do uso do big-bang com o passar do tempo e a redução no tempo de implementação dos projetos. Isso parece estar associado tanto à redução no tamanho dos projetos mais recentes como ao efeito de aprendizagem do mercado em relação ao pacote.

Conforme se assinala no texto, há uma série de considerações que devem ser feitas para a escolha do modo de início de operação, como estas: o risco que a empresa julga adequado aceitar, prazos, restrições de orçamento, a complexidade do projeto, o contexto da empresa, etc. Certamente há uma infinidade de fatores que podem interferir e que deveriam ser considerados. Nesse sentido, o presente trabalho não pretendeu criar modelo normativo da escolha, nem explicativo para a duração dos projetos. Ele apenas indica possíveis relações entre algumas variáveis direta ou indiretamente envolvidas nos projetos e eventualmente pode contribuir para o aperfeiçoamento de modelos que vierem a ser criados.

Os resultados indicam que, apesar de toda a celeuma criada em torno dos sistemas ERP e dos propalados insucessos na sua implementação, o mercado aparentemente aprendeu a pô-los em execuçaõ e a usá-los com sucesso. Os relatos sugerem e os resultados comprovam que os projetos mais antigos efetivamente tiveram mais percalços, provavelmente motivados pela falta de experiência dos implementadores nacionais e por deficiências do sistema em relação aos procedimentos e à legislação das operações comerciais brasileiras. Atualmente o sistema R/3 está sendo adotado cada vez mais por empresas menores, com menos customizações e com modo de início de operação tipo big-bang, decisão que provavelmente não seria preconizada há algum tempo. Em síntese, os resultados 
sugerem o domínio cada vez maior da tecnologia e da atividade de implementação de sistemas integrados do tipo R/3 no contexto brasileiro. Considerando que a tecnologia dos diferentes fornecedores é bastante similar, parece justo admitir que isso se aplique aos sistemas integrados de gestão em geral.

\section{Agradecimentos}

Os autores agradecem a ASUG-Brasil a oportunidade de realização da pesquisa e a colaboração recebida. Agradecimentos especiais ao Sr. Edgar Marçon e ao Sr. Fernando Birmann, membros da diretoria da ASUG-Brasil na época da realização da pesquisa.

\section{ReferênCIAs Bibliográficas}

BANCROFT, N. H.;

SEIP, H.;

SPRENGEL, A.

Implementing SAP R/3: how to introduce a large system into a large organization. 2. ed. Greenwich: Manning, 1998.

BERGAMASCHI, S.;

REINHARD, N.

Implementação de sistemas para gestão empresarial. In: ENCONTRO ANUAL DA ANPAD, 24., 2000, Florianópolis. Anais... Rio de Janeiro: ANPAD, 2000. 1 CDROM.

DAVENPORT, T. H.

Putting the enterprise into the enterprise system. Harvard Business Review, p.121-131, July/Aug. 1998.
HAIR JR., J. F. et al.

Multivariate data analysis. 5 . ed. New Jersey: Prentice Hall, 1998.

O'LEARY, D. E.

Enterprise resource planning systems: systems, life cycle, electronic commerce and risk. Cambridge: Cambridge University Press, 2000.

\section{SACCOL, A. Z. et al.}

Algum tempo depois... como grandes empresas brasileiras avaliam o impacto dos sistemas ERP sobre suas variáveis estratégicas. In: ENCONTRO ANUAL DA ANPAD, 26., 2002, Salvador. Anais...Rio de Janeiro: ANPAD, 2002. 1 CD-ROM. 
SOUZA, C. A.;

ZWICKER, R.

Implementação de sistemas ERP: um estudo de casos comparados. In: ENCONTRO ANUAL DA ANPAD, 24., 2000, Florianópolis. Anais... Rio de Janeiro: ANPAD, 2000. 1 CD-ROM.

ERP systems' life cycle: findings and recommendations from a multiple-case study in Brazilian companies. In: BALAS
ANNUAL CONFERENCE, 2001, San Diego. Proceedings... San Diego: BALAS, 2001.

WOOD, T.;

CALDAS, M.

The part and the whole: reductionism and complex thinking in ERP systems implementation. In: ENCONTRO ANUAL DA ANPAD, 24., 2000, Florianópolis. Anais... Rio de Janeiro: ANPAD, 2000. 1 CD-ROM. 